\title{
«La religion se trouvera vraie » : désenchantement et merveilles de la science chez Renan
}

\author{
"Religion will be true": Renan's Disenchantment \\ and Wonders Science
}

\author{
Azélie Fayolle \\ Université Gustave Eiffel \\ azelie.fayolle@gmail.com
}

\begin{abstract}
In 1863, the publication of Vie de Jésus makes Renan part of the movement of "desymbolisation" and secularization of sacred texts. Eliminating the notion of miracles from his writing on religion, Renan anticipates the pattern of Weber and Gauchet's "disenchantment of the world". It is thus by the methods of scientific analysis (eviction of miracles and psychological explications instead) that Renan is able to piece together the Messiah's life, putting forward the idea of an organic Parousia, and making science a new religious ideal.
\end{abstract}

Keywords: Bible, disenchantment, science, philosophy of history, miracle

Si le premier XIX ${ }^{\mathrm{e}}$ siècle lance le siècle de la critique, c'est aussi, pour FrankPaul Bowman, celui de la «désymbolisation» (Bowman, 1985). Pour Ballanche comme pour Cousin ou Strauss, il marque en effet l'entrée dans un nouvel âge de la connaissance et de la conscience, caractérisé par la réflexivité et l'abandon des symboles religieux, qu'il s'agit maintenant d'analyser et de comprendre. Cette désymbolisation passe, pour le Jeune-hégélien David-Friedrich Strauss, par « l'examen critique » de Jésus, dans Das Leben Jesu (1835, traduit par Littré en français en 1839), qui est une référence explicite de Renan pour sa propre Vie de Jésus (1863). Par le succès de cet ouvrage qui ouvre la vaste somme de l'Histoire des origines $d u$ christianisme (1863-1883), Renan devient l'ennemi à abattre : il est pour Pie IX 
«blasphémateur européen ${ }^{1}$, et son ouvrage est régulièrement interdit de lecture dans les sermons - raison probable de son immense succès. Il n'en fallait pas plus pour faire de Renan un des grands désenchanteurs du XIX siècle français, et l'auteur d'une vaste entreprise de démystification, réputation dont il s'amuse lors d'un discours prononcé en 1885 :

Moi aussi [comme plusieurs de ses aïeux], j'ai été bon taupier : j'ai détruit quelques bêtes souterraines assez malfaisantes. J'ai été un torpilleur à ma manière; j'ai donné quelques secousses électriques à des gens qui auraient mieux aimé dormir. Je n'ai pas manqué à la tradition des bonnes gens de Goëlo (1947c [1885], p. 856).

La trivialité de l'image évoque avec une légèreté dont il sera régulièrement blâmé la sécularisation opérée par son étude critique et philologique des textes sacrés : lui sont tout autant reprochés la fiction de sa réécriture, trop romanesque, que son recours aux explications physiologiques et aux sciences naturelles pour éliminer les miracles de la tradition biblique. Premier défenseur français de Darwin (Conry, 1974, p. 30), Renan reste assimilé à ce désenchantement scientiste, porté par l'essor des sciences naturelles; ces dernières lui servent bien à dissiper les brumes des mystères de la légende chrétienne. Ce désenchantement ne signe cependant pas la dissipation du sacré et de la magie que décrit d'abord Max Weber (1963, 2003), ou une sortie de la religion comme le propose Marcel Gauchet ${ }^{2}$; il ne marque pas non plus la fin de la foi, que Renan déplace, dès la rédaction de l'Avenir de la science, à une fin des temps aussi chrétienne que scientifique. Si la science, appliquée aux mythes comme au confort moderne, dissèque les légendes par la psychopathologie et décrit les saintes comme autant d'hallucinées, c'est une parousie de la connaissance que Renan espère hâter par son travail d'analyse et de critique.

\section{VIE DE JÉSUS ET DÉSENCHANTEMENT DU MONDE}

C'est peu dire que Vie de Jésus a été un choc dans le paysage littéraire et médiatique de la France de 1863, comme l'étudie Nathalie Richard (2015) dans La Vie de Jésus. Fabrique d'un best-seller. Ce succès s'explique notamment par le paradoxe d'une réécriture scientifique et dans le même temps quasi romanesque ${ }^{3}$, parce qu'élaborée comme un récit continu proche de son personnage : Vie de Jésus offre une biographie critique, reconstituée et synthétique des Évangiles. Dans la suite de Strauss, mais en s'en différenciant grandement par une approche plus historique du Christ, Renan refuse les miracles, et toute explication non rationnelle.

\footnotetext{
${ }^{1}$ D'après Jean Balcou, l'appellation date du numéro de L'Osservatore romano du 29 octobre 1872, «Pie IX vu par Renan » (voir Laurens 2013, p. 290, n. 14).

${ }^{2}$ Il ne mentionne Renan que rapidement, et avec une vue assez courte (Gauchet, 1985 p. 170).

${ }^{3}$ Le reproche revient, et est notamment analysé par Jean Kaempfer (2005).
} 
Ce sont bien les sciences naturelles ${ }^{4}$, et plus précisément l'absence de toute «trace expérimentale » (1950b [1867], p. 18) qui excluent le miracle des phénomènes admissibles par les sciences historiques. L'exceptionnelle personnalité de Jésus, « homme incomparable » (1948a [1862], p. 329), fait alors le miracle, plus que les miracles particuliers : la psychopathologie aide à la compréhension du phénomène, notamment dans le chapitre XVI intitulé "Miracles», de Vie de Jésus. Renan n'adopte pas les théories du XVIII ${ }^{\mathrm{e}}$ siècle, qui s'attachent à la recherche d'explications physiques, mais il suppose un «milieu» psychologique particulier: « Il y avait alors beaucoup de fous en Judée », écrit-il en mentionnant une « exaltation des esprits » (1950b [1867], p. 248) qui conduit à une étude des saintes, sujettes comme Christine de Stommeln, à de véritables hallucinations. Renan s'inspire alors de la psychopathologie élaborée par son collègue et proche ami, Alfred Maury, tout en historicisant davantage ses analyses. Au-delà de ces cas exceptionnels, c'est la notion de «milieu », constitué par la communauté des premiers chrétiens, qui retient Renan : il en suit l'élargissement, depuis la Galilée jusqu'à Rome, en suivant les missions des apôtres et surtout de Paul, dont le volume qui lui est consacré contient même une carte de ses déplacements. Les rapports entre les corps et les esprits forment le cœur de l'histoire renanienne : le miracle se déplace de la dérogation des lois de nature au caractère extraordinaire de ce « milieu » (1950b [1867], p. 31), fait d'attentes millénaristes et de psychologie de groupe. Si la psychopathologie retient Renan, ce n'est pas seulement pour les cas spectaculaires des hallucinations et des visions, mais pour tout ce qui permet la «propagation » du christianisme, pensé comme une fièvre - une ferveur, dont la propagation se fait selon un modèle nosologique - quoiqu'assez lâchement sollicité ${ }^{5}$.

La psychopathologie devient l'explication des miracles : de physiques, ces derniers deviennent sous la plume de Renan psychologiques. La physiologie se trouve elle aussi incluse dans ces analyses, comme quand il s'agit d'expliquer la conversion de Paul, sur la route de Damas. C'est alors sa physiologie et, peut-être, une ophtalmie qui justifie le miracle de la conversion dans Les Apôtres :

Si Paul trouva là des visions terribles, c'est qu'il les portait en son esprit. Chaque pas qu'il faisait vers Damas éveillait en lui de cuisantes perplexités. L'odieux rôle de bourreau qu'il allait jouer lui devenait insupportable. Les maisons qu'il commence à apercevoir sont peut-être celles de ses victimes. Cette pensée l'obsède, ralentit son pas ; il voudrait ne pas avancer; il s'imagine résister à un aiguillon qui le presse. La fatigue de la route, se joignant à cette préoccupation, l'accable. Il avait, à ce qu'il paraît, les yeux enflammés, peut-être un commencement d'ophtalmie. Dans ces marches prolongées, les

\footnotetext{
${ }^{4}$ En reprenant la distinction de Renan lui-même entre «sciences de la nature » et «sciences historiques », ou «sciences de l'humanité ».

${ }^{5}$ J'ai développé l'analyse de ces métaphores issues des sciences naturelles dans ma thèse Ernest Renan : savoirs de la nature et pensée historique (2019), dont j'espère la publication prochaine.
} 
dernières heures sont les plus dangereuses. Toutes les causes débilitantes des jours passés s'y accumulent; les forces nerveuses détendent, une réaction s'opère peut-être aussi le brusque passage de la plaine dévorée par le soleil aux frais ombrages des jardins détermina-t-il un accès dans l'organisation maladive et gravement ébranlée du voyageur fanatique (1950a [1866], p. 579-580).

La focalisation, interne jusqu'à la physiologie, reste prudente ; elle permet, sous couvert de scientificité, de rendre la conversion tangible pour le lecteur. Une note renforce encore ce sentiment de proximité : «J'ai éprouvé un accès de ce genre à Byblos ; avec d'autres principes, $\mathrm{j}$ 'aurais certainement pris les hallucinations que j'eus alors pour des visions » (1950a [1866], p. 580). L'attestation personnelle, repoussée en bas de page, fait de l'historien un témoin, placé devant le spectacle de l'histoire se faisant. La chose est d'autant plus sensible que la mission de Renan en Phénicie est connue, comme la mort de sa sœur Henriette sur place - Vie de Jésus lui est par ailleurs dédiée, et les circonstances de cette mort, due à une maladie qui toucha la sœur comme le frère, y sont évoquées. La physiologie comme la psychopathologie ne conduisent donc pas à un effet d'éloignement ou de distanciation, effet qui aurait pu survenir par le recours à un jargon technique. C'est au contraire vers l'hypotypose que mènent ces mentions : il s'agit d'expliquer autant que de mettre sous les yeux. Ainsi, les exaltations et les visions qui agitent, après la mort de Jésus, la croyance en sa résurrection, tiennent à des événements météorologiques pourtant quotidiens :

L'attente crée d'ordinaire son objet. Pendant un instant de silence, quelque léger souffle passa sur la face des assistants. À ces heures décisives, un courant d'air, une fenêtre qui crie, un murmure fortuit arrêtent la croyance pour des siècles. En même temps que le souffle se fit sentir, on crut entendre des sons (1950a [1866], p. 483).

Un souffle, une exaltation commune font naître et précipitent les croyances, qui se solidifient en légendes et en récits. La banalité des causes physiques, comme la narration, qui isole un moment probable - telle vision, telle conversion - pour, sur un mode itératif, le raconter comme un événement singulier mais emblématique, permettent d'inclure le lecteur dans le récit, et de lui faire sentir la vie des premières communautés chrétiennes. L' "Introduction» de Vie de Jésus rappelle d'ailleurs comment la visite de la Galilée par l'historien lui a permis d'atteindre lui-même une connaissance sensible du passé :

Toute cette histoire qui, à distance, semble flotter dans les nuages d'un monde sans réalité prit ainsi un corps, une solidité qui m'étonnèrent. L'accord frappant des textes et des lieux, la merveilleuse harmonie de l'idéal évangélique avec le paysage qui lui servit de cadre furent pour moi une révélation. J'eus devant les yeux un cinquième Évangile, lacéré, mais lisible encore, et désormais, à travers les récits de Matthieu et de Marc, au lieu d'un être abstrait, qu'on dirait n'avoir jamais existé, je vis une admirable figure humaine vivre, se mouvoir (1950b [1867], p. 79-80). 
La métaphore du « cinquième Évangile » fait du paysage un nouveau document, assimilable aux supports du philologue - Renan affirme avoir bénéficié de l'aide d'un botaniste historique pour confirmer ses intuitions (1950b [1867], p. 25). Le déplacement dans le paysage rend une réalité sensible aux textes étudiés dans la solitude des bibliothèques parisiennes ; c'est cette sensibilité que Renan rend tangible à ses lecteurs.

Permettre de toucher le passé du doigt ne constitue cependant pas un acte littéraire anodin : cette réécriture permet une appropriation personnelle et sentimentale $\mathrm{du}$ Christ, encore renforcée par la parution de l'édition populaire, Jésus, destinée à un public plus large. Si Renan se défend de tout manquement au dogme, c'est justement par l'accessibilité au Christ et à ses premiers - et ses premières - fidèles, qu'il effectue un véritable acte, non de profanation, mais de désacralisation, qui est aussi, par éviction des miracles, un désenchantement.

\section{DÉSENCHANTEMENT DE LA SCIENCE ET PROGRÈS}

On aurait pu croire que Renan, « tueur de mythes » à l'image d'un Max Müller ${ }^{6}$, serait un chantre du progrès technique. Malgré son titre, l'article «La Poésie de l'exposition » contredit cette idée : c'est au contraire l'absence de toute poésie du progrès appliqué qui fait le fond de cette diatribe, qui a valu à Renan quelques réponses courroucées des lecteurs du Journal des Débats. Le philosophe n'assimile jamais les progrès matériels à ceux de la science ou de l'esprit ; c'est au contraire un abaissement moral qui résulterait des améliorations de la vie moderne :

Une civilisation complète doit tenir compte de l'art et de la beauté presque autant que de la morale et du développement intellectuel. Mais, loin que les progrès de l'art soient parallèles à ceux que fait une nation dans le goût du confortable (je suis obligé de me servir de ce mot barbare pour exprimer une idée peu française), il est permis de dire sans paradoxe que les temps et les pays où le confortable est devenu le principal attrait du public ont été les moins doués sous le rapport de l'art (1948c [1855], p. 242).

L'emprunt se sent encore : le confortable signe un avilissement moral, mais il n’élève pas («n'ennoblira pas » [Renan, 1948b [1855], p. 248]). Il n’est pas alors anodin de considérer que Renan, en reprenant l'article dans son volume Essais de morale et de critique (1948b [1859]), le place avant «La Poésie des races celtiques » (1948d [1854]) qui en constitue le contre-modèle. Les femmes sont, dans un texte comme dans l'autre, la raison de cette absence (parce que leur goût pour le luxe amoindrirait les hauts instincts, dans un souvenir rousseauiste) ou de cette présence de poésie. La réduction du progrès industriel aux arts ménagers montre par

\footnotetext{
${ }^{6}$ Je reprends cette formule à Bertrand Marchal (2015).
} 
ailleurs la séparation radicale que Renan opère entre science appliquée et science fondamentale.

La science peut bien désenchanter les origines du christianisme ; détachée des progrès technologiques, elle n'est même pas mentionnée comme une origine possible des améliorations quotidiennes, que Renan finit par promouvoir. Le désenchantement provoqué par l'analyse des mythes ne trouve pas son pendant dans le progrès technique, lumière artificielle ou lumière électrique, tout au contraire : la science est en elle-même un enchantement, quand ses applications pratiques (et populaires) n'ont aucune importance véritable. Aussi, au banquet « Scientia » organisé pour honorer son vieil ami, le chimiste Marcelin Berthelot, Renan peut-il rappeler ce stéréotype d'une science désenchanteresse, pour s'en moquer :

J'estime donc très peu fondée l'éternelle jérémiade de certains esprits sur les prétendus paradis dont nous prive la science. Nous savons plus que le passé ; l'avenir saura plus que nous. Vive l'avenir! Vous aurez largement contribué, cher ami, à ce progrès de l'esprit, où la part de notre siècle, quoi qu'on dise, sera belle. Dans la plus philosophique peut-être des sciences, la chimie, vous avez porté les limites de ce que l'on sait au delà du point où s'étaient arrêtés vos devanciers. Dilater le pomoerium, c'est-à-dire reculer l'enceinte de la ville était, à Rome, l'acte de mémoire le plus envié. Vous avez dilaté, mon cher ami, au secteur où vous travaillez, le pomoerium de l'esprit humain (1947b [1885], p. 861).

L'optimisme de Renan se trouve tout entier dans cet appel à l'avenir, qu'il répète dans ces discours de la fin de sa vie. Sans partager l'émerveillement de l'abbé Pluche du Spectacle de la nature, Renan déplace sa foi chrétienne, perdue à sa sortie du séminaire, en 1845, en une foi laïque et scientifique.

\section{VERS UNE PAROUSIE SCIENTIFIQUE : L’ÉMERVEILLEMENT À VENIR}

L'entrée dans ce que Renan appelle l' ' âge de l'analyse » se fait par l'ouverture d'une période scientifique. C'est d'abord la dissipation des vieilles chimères qui signale l'entrée dans ce nouveau paradigme : «On ne reconstituera pas les anciens rêves », écrit Renan dans son «Examen de conscience » de 1890 (1947d [1890], p. 1179). Si Renan se plaît à imaginer l'avenir, ce n'est pas, malgré les « Rêveries » des Dialogues philosophiques (1947a [1876]), des rêves que doit produire la science, mais une vérité encore intangible - le rêve futur n'est que l'attente d'une réalité encore non advenue. Dans le temps présent, la science donne à penser, comme le suggèrent les conversations des commensaux des dîners Magny et Brébant, hypothèses et parfois plaisanteries sur l'avenir, mais elle permet déjà un choc esthétique celui de l'«éclat» des paroles du Christ, qui se « décèlent pour ainsi dire d'ellesmêmes » (1950b [1867], p. 79 et 80), celui des images tout aussi frappantes qu'offre 
la science, comme le montre le portrait de Claude Bernard, devenu un véritable augure. Les résultats scientifiques permettent eux-mêmes un émerveillement :

Le calcul infinitésimal ne roule assurément que sur des formules ; mais ces formules sont des symboles frappants. Il y a des ordres divers d'infini, dont les inférieurs sont zéro à l'égard des supérieurs. Ce paradoxe apparent sert de base à des calculs d'une absolue vérité. Toute quantité finie, ajoutée à l'infini ou retranchée de l'infini, équivaut à zéro ; toute quantité finie n'est rien comparée à l'infini. Nos idées de l'espace et du temps sont toutes relatives. La distance de la terre à Sirius est énorme d'après nos mesures. Les vides intérieurs d'une molécule peuvent être aussi considérables pour des êtres doués d'un autre critérium de la grandeur. La longévité de notre monde pourrait, aux yeux d'un dieu, paraître l'équivalent d'un jour (1949 [1848, 1890], p. 1165).

Les réflexions sur les deux infinis, inspirées, quoique lointainement du calcul infinitésimal de Leibniz, sont propres à retrouver l'effroi pascalien. Sous la plume de Renan, les formules mathématiques deviennent des « symboles frappants » : la locution, si elle peut apparaître anodine, noue une philosophie de l'histoire du langage et l'émerveillement esthétique. L'âge du symbole, normalement attribué à l'époque d'une humanité primitive, devient celui de l'âge de l'analyse : le symbole, phase syncrétique de la pensée, se retrouve par la science. La locution rassemble autant l'épistémologie renanienne du devenir qu'une réception esthétique, qui accueille le regard du novice comme celui du savant qui saura déchiffrer ces symboles. Renan suppose en effet une histoire pensée, à partir de la scolastique, comme un fieri, et qu'il présente comme mue, en termes naturalistes, par une force qu'il qualifie de nisus : il retrouve là les termes du naturaliste Blumenbach, qui suppose dans la formation de l'embryon un nisus formativus. Cette force organique individuelle devient, sous la plume de l'historien, force historique : elle emporte dans son mouvement l'ensemble de l'univers, depuis un syncrétisme primitif jusqu'à une analyse provisoire (et contemporaine), qui doit conduire à un nouvel âge de la synthèse. Ces catégories, probablement inspirées de la philosophie cousinienne, se retrouveraient dans la formation des organismes matériels comme immatériels, les langues et les religions, ou l'univers pris dans son ensemble.

Parce qu'elle est téléologique, la science telle que la pense Renan n'épuise pas l'émerveillement: elle en élargit l'application et le renouvelle. La préface de L'Avenir de la science, rédigé en 1848 mais publié seulement en 1890, en porte la trace, en rappelant qu' « il n'y a guère plus d'un siècle que la raison travaille avec suite au problème des choses. Elle a trouvé des merveilles, qui ont prodigieusement multiplié le pouvoir de l'homme » (1949 [1848, 1890], p. 728), sans qu'il précise en quoi consiste ce «pouvoir». C'est une véritable parousie que suppose Renan, supposition affichée dans sa lettre, adressée à Marcelin Berthelot, « Les sciences de la nature et les sciences historiques »: «Ce triomphe de l'esprit, ce vrai royaume de Dieu, ce retour au modèle idéal me semblent la fin suprême du monde » (1947e [1863], p. 648). La reprise explicite de la formule évangélique ouvre une véritable 
foi scientiste, qui conserve les contours et les formes du christianisme pour produire une parole quasi assimilable au logos divin. Si le passage reste prudent, la suite de cette lettre se fait toute prophétique :

Alors nous régnerons, nous tous hommes de l'idée. Nous serons cendres depuis des milliards d'années, les quelques molécules qui font la matière de notre être seront désagrégées et passées à d'incalculables transformations; mais nous ressusciterons dans le monde que nous aurons contribué à faire. Notre œuvre triomphera. Le sens moral alors se trouvera avoir eu raison; la foi, qui croit contre l'apparence, sera justifiée : c'est elle qui aura bien deviné ; la religion se trouvera vraie (1947e [1863], p. 648).

La disparition progressive des modalisateurs opère non seulement le passage de l'hypothèse à la prophétie, mais aussi la fusion entre savoir et croire, entre science et religion - ce sont des «molécules » qui croisent les cendres de l'Ecclésiaste, et qui confirmeront scientifiquement les textes bibliques.

Si la fin des temps se pense, pour Renan, comme une parousie, celle-ci advient déjà sous sa plume. À ce titre, l'embryogénie de l'esprit humain qu'il se propose d'ériger en but de ses travaux dès ses premiers textes, comme De l'Origine du langage (1958 [1848]), n'est pas à comprendre comme une simple métaphore, mais comme un modèle de pensée qui nourrit la méthodologie de ses textes historiens. Il s'agit pour Renan de revenir aux temps des origines (temps « embryonnaires ») en appliquant des méthodes inspirées des sciences naturelles, comme l'analogie et le raisonnement par induction; l'embryon à l'origine du projet historien ne peut alors plus, si l'on suit les théories de Max Black (voir 1962), être considéré comme une simple métaphore, mais il est, en raison de son étayage théorique, un modèle de pensée.

L' "Introduction » de Vie de Jésus permet à Renan de revenir sur cet organicisme qui sous-tend le projet d'une embryogénie de l'esprit humain :

Une grande vie est un tout organique qui ne peut se rendre par la simple agglomération de petits faits. Il faut qu'un sentiment profond embrasse l'ensemble et en fasse l'unité. [...] Les lois intimes de la vie, de la marche, des produits organiques, de la dégradation des nuances, doivent être à chaque instant consultées ; car ce qu'il s'agit de retrouver, ce n'est pas la circonstance matérielle, impossible à vérifier, c'est l'âme même de l'histoire ; ce qu'il faut rechercher, ce n'est pas la petite certitude des minuties, c'est la justesse du sentiment général, la vérité de la couleur. Chaque trait qui sort des règles de la narration classique doit avertir de prendre garde ; car le fait qu'il s'agit de raconter a été conforme à la nécessité des choses, naturel, harmonieux (1950b [1867], p. 81).

Les sciences naturelles participent ainsi de la constitution du récit historien, et en particulier du récit biographique. Renan va plus loin que ce « sentiment» quelque peu général : « Ce sentiment d'un organisme vivant », poursuit-il, « on n'a pas hésité à le prendre pour guide dans l'agencement général du récit » (1950b [1867], p. 81-82). 
Si un certain « sentiment », complémentaire de la "part [permise] de divination et de conjectures » (1950b [1867], p. 81), guide le récit jusqu'à sa structuration, c'est aussi le style classique, tel qu'hérité d'une langue du XVII ${ }^{\mathrm{e}}$ siècle quelque peu fantasmée, qui se retrouve dans cet idéal à la fois harmonieux et naturel, mais aussi naturaliste la science se retrouve, comme injectée, dans le moule de la langue classique.

\section{CONCLUSION}

«Un peu de science en éloigne, beaucoup y ramène »; Renan pourrait correspondre à une de ces idées reçues du dix-neuvième siècle, recueillies par Flaubert et ici portée par le comte de Faverges. La banalité de la formule occulterait cependant la profondeur de l'émerveillement contemplatif, comme l'ironie du vieux Renan. Éternel prêtre marqué par un «pli ineffaçable » (1948e [1883], p. 892), Renan ne répète pas son missel : il réécrit les textes bibliques en en élargissant la portée - sous sa plume, le texte évangélique se fait scientifique. L'image du «taupier» dont s'amuse l'historien en 1885 trouve dans ce travail de la langue sa réalisation. En supposant, en regard des produits naturels, des « produits de l'esprit humain », pensé selon un modèle organiciste peu remis en question dans les textes publiés, Renan n'est pas seulement l'agent d'une sécularisation, comprise par Weber ou Gauchet comme un «désenchantement»; il reprend plutôt les « anciens rêves » pour leur donner une réalité nouvelle. Il n'y a pas, dans la perspective renanienne, de désenchantement véritable, mais de nouveaux modes d'enchantement.

La vie et le vivant vont fournir les modèles de pensée de cette philosophie de l'histoire. C'est le développement des corps organiques, plus que l'évolution des espèces, et leur mort seulement apparente, autant par la dispersion atomique que par la permanence des idées, qui servent de parangons aux théories et aux textes de l'historien. À ce titre, le propos de Renan est radicalement opposé aux thèses de Marcel Gauchet quand il affirme que les dieux «survivent, c'est leur puissance qui meurt » (Gauchet, 1985, p. IV) ; Renan pour sa part voit dans la religion le « résumé des besoins moraux de l'homme, la vertu, la pudeur, le désintéressement, le sacrifice », toutes choses qui « sont la voix de l'univers » (1947d [1889], p. 1174), alors anthropomorphisé. Plutôt que politique ou sociale, la religion est alors constituante de la nature humaine :

Tout se résume en un acte de foi à des instincts qui nous obsèdent, sans nous convaincre, en l'obéissance à un langage parfaitement clair en ce qu'il nous commande, obscur en ce qu'il promet. Nous voyons le charme ; nous le déjouons ; mais il ne sera jamais rompu pour cela. Quis posuit in visceribus hominis sapientiam? (1947d [1889], p. 1174)

Autrement dit : les dieux meurent (ou ne sont plus écoutés), quand leur puissance (leur charme) persiste - viscéralement. 


\section{BIBLIOGRAPHIE}

Black, M. (1962). Models and Metaphors. Studies in Language and Philosophy. Ithaca: Cornell University Press.

Bowman, F.-P. (1985). Symbole et désymbolisation. Romantisme, 50, 53-60. URL : https://www. persee.fr/doc/roman_0048-8593_1985_num_15_50_4752

Conry, Y. (1974). L'Introduction du darwinisme en France au XIX ${ }^{e}$ siècle. Paris : Vrin.

Gauchet, M. (1985). Le Désenchantement du monde. Une histoire politique de la religion. Paris : Gallimard.

Kaempfer, J. (2005). La Vie de Jésus, tout un roman... Études de lettres, 3, 37-49. URL : https:// www.unil.ch/usagesdejesus/files/live/sites/usagesdejesus/files/shared/La_vie de Jesus.pdf

Laurens, H. (Éd.) (2013). La Science, la religion, la République. Paris : Odile Jacob. Travaux du Collège de France.

Marchal, B. (2015). Max Müller. Le tueur de mythes, suivi de Notes sur Flaubert et Müller. In P.-M. de Biasi, A. Herschberg Pierrot \& B. Vinken (Éds.), Flaubert. Les pouvoirs du mythe. T. 1 (pp. 41-56). Paris : Éditions des Archives Contemporaines.

Renan, E. (1947a [1876]). Dialogues philosophiques. In Euvres complètes. T. I (pp. 547-632). Éd. H. Psichari. Paris : Calmann-Lévy.

Renan, E. (1947b [1885]). Discours à la conférence «Scientia», Banquet en l'honneur de M. Berthelot. In Euvres complètes. T. I (pp. 859-861). Éd. H. Psichari. Paris : Calmann-Lévy.

Renan, E. (1947c [1885]). Discours prononcé à Quimper. In Euvres complètes. T. I (pp. 853-858). Éd. H. Psichari. Paris : Calmann-Lévy.

Renan, E. (1947d [1889]). Examen de conscience philosophique. In Euvres complètes. T. I. Éd. H. (pp. 1162-1182). Psichari. Paris : Calmann-Lévy.

Renan, E. (1947e [1863]). Les sciences de la nature et les sciences historiques. In Euvres complètes. T. I (pp. 633-650). Éd. H. Psichari. Paris : Calmann-Lévy.

Renan, E. (1948a [1862]). De la part des peuples sémitiques dans l'histoire de la civilisation. Discours d'ouverture du cours de langues hébraïque, chaldaïque et syriaque au Collège de France. In Euvres complètes. T. II (pp. 317-335). Éd. H. Psichari. Paris : Calmann-Lévy.

Renan, E. (1948b [1859]). Essais de morale et de critique. In CEuvres complètes. T. II (pp. 11-301). Éd. H. Psichari. Paris : Calmann-Lévy.

Renan, E. (1948c [1855]). La Poésie de l'exposition. In CEuvres complètes. T. II (pp. 239-251). Éd. H. Psichari. Paris : Calmann-Lévy.

Renan, E. (1948d [1854]). Poésie des races celtiques. In Euvres complètes. T. II (pp. 252-301). Éd. H. Psichari. Paris : Calmann-Lévy.

Renan, E. (1948e [1883]). Souvenirs d'enfance et de jeunesse. In CEuvres complètes. T. II (pp. 713-931). Éd. H. Psichari. Paris : Calmann-Lévy.

Renan, E. (1949 [1848, 1890]). L'Avenir de la science. In Euvres complètes. T. III (pp. 11-123). Éd. H. Psichari. Paris : Calmann-Lévy.

Renan, E. (1950a [1866]). Les Apôtres. In CEuvres complètes. T. IV (pp. 433-702). Éd. H. Psichari. Paris : Calmann-Lévy.

Renan, E. (1950b [1867]). Vie de Jésus. In Euvres complètes. T. IV (pp. 11-427). Éd. H. Psichari. Paris : Calmann-Lévy.

Renan, E. (1958 [1848]). De l'origine du langage. In Cuvres complètes. T. VIII (p. 715-1121). Éd. H. Psichari. Paris : Calmann-Lévy.

Richard, N. (2015). La Vie de Jésus de Renan. La fabrique d'un best-seller. Rennes: Presses Universitaires de Rennes.

Weber, M. (1963). Le Savant et le politique. Paris : Plon.

Weber, M. (2003). L'Éthique protestante et l'esprit du capitalisme. Suivi d'autres essais. Paris : Gallimard. 